\title{
An updated description of heavy-hadron interactions in GEANT-4
}

\author{
R. Mackeprang ${ }^{1}$, D.A. Milstead ${ }^{2, a}$ \\ ${ }^{1}$ CERN, Geneva, Switzerland \\ ${ }^{2}$ Fysikum, Stockholms Universitet, Stockholm, Sweden
}

Received: 1 September 2009 / Revised: 26 November 2009 / Published online: 17 February 2010

(C) Springer-Verlag / Società Italiana di Fisica 2010

\begin{abstract}
Exotic stable massive particles (SMP) are proposed in a number of scenarios of physics beyond the Standard Model. LHC experiments are expected to be able both to detect and extract the quantum numbers of any SMP with masses around the $\mathrm{TeV}$ scale. An understanding of the interactions of SMPs in matter is required to optimise the detection methods and calculate acceptances in an SMP search. In this paper a regge-based model of $R$-hadron scattering is extended and implemented in GEANT-4. In addition, the implications of $R$-hadron scattering for collider searches are discussed.
\end{abstract}

\section{Introduction}

The observation of an exotic stable ${ }^{1}$ massive particle (SMP) with electric, magnetic or colour charge (or combinations thereof) would be of fundamental significance. Searches are therefore routinely made for SMPs bound in matter, and for SMPs at cosmic ray and collider experiments [1-5]. Furthermore, SMPs with masses at the $\mathrm{TeV}$ scale are anticipated in a number of beyond-the-Standard-Model scenarios, such as supersymmetry and universal extra dimensions [4, 5]. SMP searches will thus be a key component of the early data exploitation programme of the LHC experiments [6-8]. To facilitate such searches, models are needed of the interactions of SMPs as they pass through matter. Although robust phenomenological approaches are available to predict the scattering of electromagnetically charged SMPs, there is no consensus on how the interactions of hadronic SMPs (hereafter termed $R$-hadrons ${ }^{2}$ ) should be treated. This is due to un-

\footnotetext{
${ }^{1}$ The term stable is taken to mean that the particle will not decay during its traversal of a detector.

${ }^{2}$ The term $R$-hadron has its origin in the $R$-parity quantum number in supersymmetric theories and the work in this paper will be presented in the context of supersymmetric particles. However, the results given here are generally applicable to stable heavy exotic hadrons.

a e-mail: milstead@physto.se
}

certainties associated with the strong scattering processes and the hadronic mass hierarchy. In this work a model of $R$-hadron scattering [9] which is based on triple regge phenomenology [10] is extended and implemented in GEANT4 [11]. Although the main purpose of this work is to aid searches at colliders, it is also envisaged that it will be useful for cosmic-ray searches [12].

Hadronic interactions of $R$-hadrons at colliders can give rise to energy loss and a range of striking signatures. Scattering processes can thus potentially enhance the discovery capability of an experiment as well as challenging it's eventreconstruction algorithms [4]. For example, due to scattering effects, an SMP can possess different values of electric charge as it traverses a detector. Furthermore, hadronic interactions affect the rate at which SMPs lose energy and thus the potential of experiments to discover SMPs via the late decays of stopped SMPs [13, 14].

To aid the development of search strategies, it is thus important that experiments have access to Monte Carlo simulation models which span as fully as possible the range of conceivable signatures which could be associated with $R$ hadron production. At present, one model is available within GEANT-4 [15]. This model, hereafter termed the generic model, is based on a black-disk approximation [16] and employs pragmatic assumptions regarding possible scattering processes and stable $R$-hadron states. The work presented in this paper concerns an approach, hereafter termed the triple regge model, which uses the triple regge formalism to predict the scattering of $R$-hadrons formed from stop-like $(\tilde{t})$ and sbottom-like $(\tilde{b})$ exotic colour-triplet squarks $(\tilde{q})$ and electrically neutral gluino-like ( $\tilde{g})$ colour-octet particles [9]. Furthermore, the model uses well motivated assumptions regarding $R$-hadron mass hierarchies which differ from those employed by the generic model.

This paper is organised as follows. First, we discuss mass hierarchies of $R$-hadrons. This is followed by a description of the triple regge model and its implementation in GEANT4. Finally, model calculations of the energy loss and flavour 
composition for $R$-hadrons passing through iron are shown and discussed.

\section{Exotic heavy hadrons and their masses}

Fermionic mesons $\left(\tilde{q} \bar{q}^{\prime}, \tilde{g} q \bar{q}^{\prime}\right)$ and bosonic baryons $\left(\tilde{q} q^{\prime} q^{\prime \prime}\right.$, $\tilde{g} q q^{\prime} q^{\prime \prime}$ ) would be expected to be formed from stable squark and gluino states, where $q, q^{\prime}$ and $q^{\prime \prime}$ denote Standard Model quarks [17]. ${ }^{3}$ In this work, with the exception of the lightest gluino baryonic state (described below), only $R$-hadrons comprising light valence quarks of type $u$ and $d$ are considered.

Calculations of $R$-hadron mass spectra have been made using a variety of approaches such as the bag model [1820] and lattice QCD [21]. These predictions, together with measurements of heavy hadron masses, make it possible to approximately determine those features of the mass hierarchies which are most relevant for the modelling of $R$-hadron scattering in matter. Of particular interest are the masses of the lowest-lying states to which higher mass particles would be expected to decay before interacting hadronically and the mass splitting between the lightest meson and baryon states.

Given the observed spin and flavour independence of interactions of heavy quarks with light quarks [22-24], the lightest squark-based meson and baryon states can be inferred [25] from the measured mass spectra of charm and bottom hadrons. Thus, using the mass spectra of the observed charm and bottom mesons as a guide, charged and neutral squark-based mesons would be approximately mass degenerate, and the lightest baryon state would be the scalar singlet $\tilde{q} u d$. This would be the exotic equivalent of the $\Lambda_{c}$, $\Lambda_{b}$ baryons. This picture is supported by calculations of hyperfine splittings of a few hundred $\mathrm{MeV}$ between the $\tilde{q} u d$ state and the vector states $\tilde{q} u u, \tilde{q} d d$ and $\tilde{q} u d[16,25]$. Furthermore, the mass difference between the stable baryon and mesonic states would be far less than $1 \mathrm{GeV}$ and the decay of a baryon into a meson and proton would be kinematically forbidden [16, 25].

The basic properties of gluino $R$-hadrons may not be similarly inferred from Standard Model hadronic mass spectra. A greater reliance on phenomenological approaches is therefore required to identify the stable states. Possible hadrons include gluino $R$-mesons $\left(\tilde{g} q \bar{q}^{0}, \tilde{g} u \bar{d}^{+}, \tilde{g} d \bar{u}^{-}\right)^{4}$ and gluinogluon states $(\tilde{g} g)$. Various calculations, such as in Ref. [18] (bag model), Ref. [21] (lattice QCD) and Ref. [16] (Potential model) estimate the masses of the gluino $R$-mesons to be the gluino mass plus $0.2-0.7 \mathrm{GeV}$. The mass differences between the lowest lying states of each of these hadrons are

\footnotetext{
${ }^{3}$ Where no ambiguity is likely to arise, a reference to a particle is also taken to refer to its antiparticle in this paper.

${ }^{4}$ Here $q \bar{q}$ represents a mixture of $u \bar{u}$ and $d \bar{d}$-states.
}

less than the pion mass. Calculations of the lowest lying baryonic states using the bag model $[19,20]$ predict that the lightest state is the flavour singlet $\tilde{g} u d s^{0}$, and that the "nucleon" $R$-baryons $\left(\tilde{g} u d d^{0}, \tilde{g} u u d^{+}\right)$are $0.2-0.3 \mathrm{GeV}$ heavier. The consequence of this is that even though these heavier states would be stable to strong decays, they would decay weakly into the singlet state over a time scale of $\sim 10^{-10} \mathrm{~s}$. Although this is most likely for a low mass gluino hypothesis $[19,20]$, in view of the uncertainties associated with $R$ hadron propagation through matter, this possibility is considered in this work since it leads to an experimentally challenging $R$-hadron signature at a collider. The mass of the $\tilde{g} u d s^{0}$ state is typically in the range of the gluino mass plus $0.2-0.7 \mathrm{GeV}[18,20]$. It is thus reasonable to assume that all gluino $R$-hadrons would be approximately degenerate in mass. Specifically none of the models allow for an $R$ meson/ $R$-baryon mass splitting larger than the proton mass thus disallowing decays from baryonic to mesonic states.

For the regge model, we use the squark and gluino $R$ hadron mass hierarchy assumptions given in Table 1 . In addition, to avoid attempting calculations of poorly understood cascade decays, we use simplified mass spectra which comprise only those $R$-hadrons deemed to be stable. The underlying assumption is that any higher states would decay to the stable states before having an opportunity to interact. The generic model uses a an approach in which members of all hadron multiplets with light quarks are degenerate, and thus stable.

Partly as a consequence of the assumed stable states, $R$ hadron event topologies can be anticipated which would be challenging for one of the most common collider search methods. The efficiencies of searches for slow muon-like objects $[6,26-30]$ would be affected by the $R$-baryon production processes which take place as $R$-hadron pass through matter. As discussed further in Sect. 3, such processes imply that a sbottom or gluino $R$-hadron, irrespective of its state as it entered a calorimeter, is likely to leave as an uncharged object and therefore escape detection in an outer muon detector.

Although well motivated mass-hierarchy assumptions were selected for this work, in the absence of experimental data, alternative scenarios should also be considered.

Table 1 Assumed stable hadrons formed from squarks $(\tilde{q})$ and gluinos $(\tilde{g})$, together with the values of masses used in this work. The neutral gluino states containing a mixture $u \bar{u}$ and $d \bar{d}$ pairs are generically denoted by $\tilde{g} q \bar{q}$

\begin{tabular}{lll}
\hline Heavy Parton & States & Mass $(\mathrm{GeV})$ \\
\hline Squark & $\tilde{q} \bar{u}, \tilde{q} \bar{d}$ & $m_{\tilde{q}}+0.3$ \\
& $\tilde{q} u d$ & $m_{\tilde{q}}+0.7$ \\
\multirow{2}{*}{ Gluino } & $\tilde{g} q \bar{q}, \tilde{g} u \bar{d}, \tilde{g} d \bar{u}, \tilde{g} g$ & $m_{\tilde{g}}+0.7$ \\
& $\tilde{g} u d s$ & $m_{\tilde{g}}+0.7$ \\
\hline
\end{tabular}


The generic $R$-hadron scattering model makes more pragmatic assumptions regarding the mass hierarchies. In particular, it assumes degenerate stable squark (gluino) baryon states: $\tilde{q} u d, \tilde{q} d d, \tilde{q} u u$. ( $\tilde{g} u u d, \tilde{g} u d d, \tilde{g} d d d, \tilde{g} u u u)$. This would lead to the bulk of sbottom and gluino $R$-hadrons being charged after they leave a typical calorimeter [6]. Thus, taken together, the two models will aid in the development of a more complete set of collider searches for $R$-hadrons.

In this paper, the stable states in Table 1 are exceptionally used with the generic model in order to study the contribution of the different factors (scattering effects and stable mass states) to predictions of energy loss and transformation of $R$-hadrons in matter.

\section{Scattering of $\boldsymbol{R}$-hadrons in matter}

Although the fine details of $R$-hadron scattering are difficult to model, first-principle arguments can be used to build up a qualitative picture of the processes through which $R$ hadrons suffer energy loss and undergo charge and baryon number exchange.

The probability of an interaction between the heavy parton and a quark in the target nucleon is low since the cross section varies with the inverse square of the parton mass according to perturbative QCD [16]. One can thus use a picture of scattering of a stable non-interacting heavy parton accompanied by a coloured hadronic cloud of light constituents which take part in the scattering. Energy losses of an $R$-hadron in an interaction with a stationary nucleon will thus be determined by the kinetic energy of the light quark system in an $R$-hadron. At the LHC, for $R$-hadrons of masses above several hundre $\mathrm{GeV}$ the light quarks system's kinetic energy is typically of $\mathcal{O} 1 \mathrm{GeV}$ and small energy losses are thus anticipated. Another important feature of $R$ hadron scattering is that processes in which $R$-baryons are converted into $R$-mesons are suppressed due to kinematics and the absence of pions in material [16]. Thus, any mesons which convert to baryons will likely stay in this state during their passage through material. A number of models have been proposed $[9,15,16,31,32]$ which are based on the above principles. In this section, brief summaries are given of the salient features of the triple regge [9] and generic models $[15,16]$. This is followed by a description of the implementation of the triple regge model in GEANT- 4 .

An alternative model of scattering involves the $R$-hadron being stripped of it's light quark system and then being forced to refragment [31]. The properties of the transformed $R$-hadron would be prescribed by free parameters. In this picture, the above arguments concerning baryon conversion may not apply. It should, however, be noted that the two extremes cases represented by the regge and generic models would approximately cover the range of $R$-hadron signatures expected in this scenario.

\subsection{Triple regge model}

Since the central picture used is one of a low-energy lightquark system interacting with a stationary nucleon, $R$ hadron scattering can be treated with the phenomenology used to describe low-energy hadron-hadron scattering data [9, 31, 32], as is done in the triple regge approach [9]. The triple regge model was originally developed to describe the scattering of exotic hadrons containing heavy colourtriplet objects. Here, though, it has been extended to also treat gluino $R$-hadrons. This model assumes the stable states described in Sect. 2.

Using parameters fitted to low-energy hadron-hadron data, the triple regge model makes predictions for $R$-hadron scattering cross sections, together with energy-loss calculations based on the triple regge formalism. Figure 1 (top) shows the model predictions of the scattering cross sections of a squark-based $R$-hadron off a stationary nucleon within a nucleus comprising equal numbers of neutrons and protons. The cross section formulae are given in Sect. 3.3 in which the implementation of the model in GEANT-4 is described. The cross section is shown for different types of squark-based $R$-hadrons as a function of the Lorentz factor, $\gamma$. As can be seen, there is a large cross section for antibaryon $(\overline{\tilde{q}} \bar{u} \bar{d})$ interactions which is due to a dominant annihilation process with a nucleon in the target.

It is also seen that, at lower values of $\gamma(\gamma \lesssim 10)$, the scattering cross section of squark-based $R$-hadrons containing a light valence antiquark $(\tilde{q} \bar{u}, \tilde{q} \bar{d})$ is larger than for antisquark-based $R$-mesons. This arises from the presence of reggeon-exchange processes which are only permitted for $R$-hadrons containing a light antiquark. Owing to the presence of an additional light quark the scattering cross section for $R$-baryons ( $\tilde{q} u d)$ is twice as large as for the mesons with light quarks.

Upon an interaction the probability that an $R$-meson becomes a baryon is $10 \%$. Once an $R$-hadron becomes a baryon it stays in this state. Another process which must be taken into account is the oscillation of a neutral mesonic squark-based $R$-hadron into its antiparticle [25, 33]. Feynman diagrams of possible processes in which oscillations can occur are shown in Fig. 2. Tree level gaugino (gluino and neutralino) exchange and one-loop charged current-chargino box diagrams are shown. Since the conversion rate would be model dependent we allow two possibilities here: zero mixing, in which no oscillations take place and a maximalmixing scenario in which there is a $50 \%$ probability that any neutral mesonic squark-based $R$-hadron which was produced would automatically be converted to its anti-particle. These mixing scenarios correspond to oscillation lengths which are infinite and zero, respectively.

In Fig. 1 (bottom) the predicted cross sections for gluino $R$-hadron species are shown. The baryon cross section is 

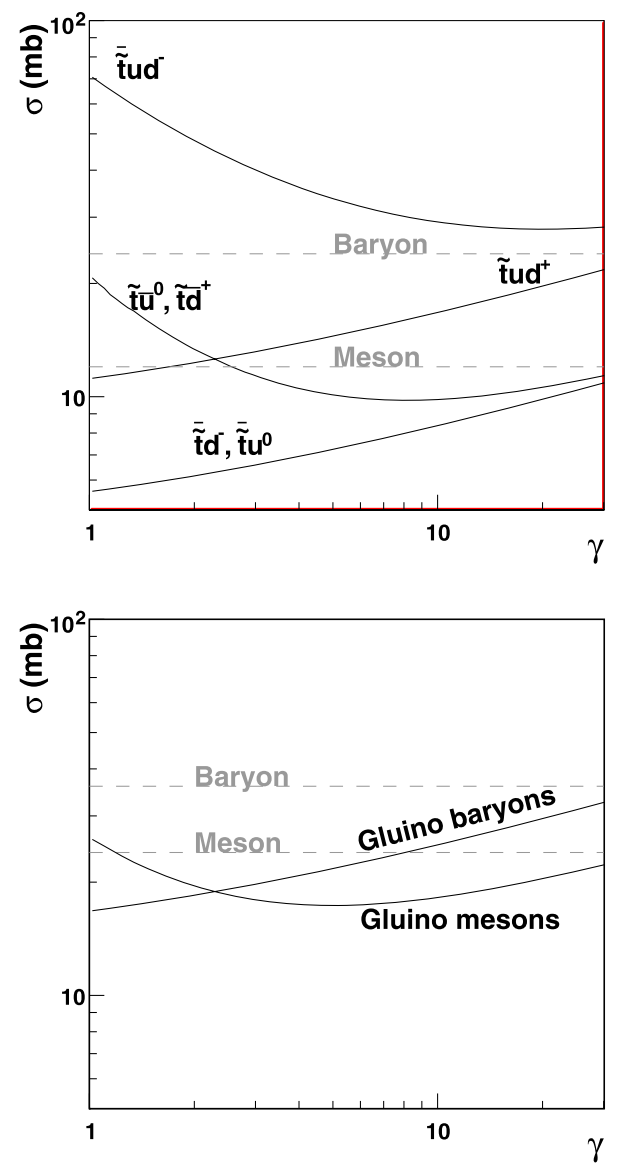

Fig. 1 Cross sections for the interaction of stop-based and gluino-based $R$-hadrons off a stationary nucleon within a nucleus assumed to contain an equal number of protons and neutrons. The predictions are shown for the triple regge model (solid lines) and the generic model (dashed lines)
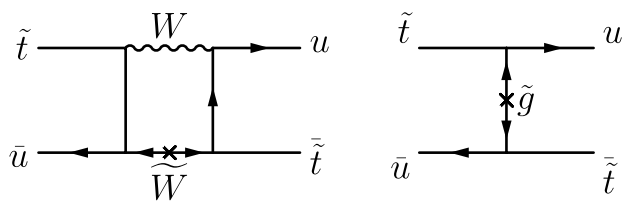

Fig. 2 Feynman diagrams representing oscillations of neutral stop-based $R$-mesons

$50 \%$ greater than that for stop $R$-hadrons owing to the extra light quark in the gluino $R$-hadron. Similarly, since the gluino meson contains a light quark and antiquark, then both regge and pomeron-exchange processes are available. The scattering cross section for gluino mesons at a fixed value of $\gamma$ is the sum of the cross sections for a stop meson and anti-stop meson. Following Ref. [16], a gluino-gluon state is assumed to scatter in the same way as a gluino-based $R$ meson. Again, a baryon number transfer probability of $10 \%$ is used.

\subsection{Generic model}

The generic model is already available in GEANT-4 [15] and is an update of earlier work [16]. In view of the inherent uncertainties associated with modelling $R$-hadron scattering a pragmatic approach is assumed in which the scattering rate is estimated with a constant geometric cross-section of $12 \mathrm{mb}$ per light quark. All 2-to-2 and 2-to-3 processes are allowed if they are kinematically feasible and charge conservation is respected. The proportion of 2-to-2 and 2-to-3 reactions is governed by phase space factors while no explicit constraints are applied to the probability of baryon number exchange. The constant cross sections for stop and gluino $R$-meson and $R$-baryon interactions are superimposed on Fig. 1 (top and bottom). This model assumes $R$-hadron mass hierarchies which differ from those used in the triple regge model and predicts that the majority of baryons which are produced in scattering processes are electrically charged.

\subsection{Implementation of triple regge model in GEANT-4}

In the dynamical picture of $R$-hadron scattering used by GEANT, the light quark system is decoupled from the heavy spectator parton before interacting with a nucleon according to GEANT's parameterised model for light hadrons. In this way secondary particles and so-called black tracks are generated. Following the interaction, the light-quark system is recombined with the heavy parton.

To implement the triple regge model in Geant-4, the software architecture used for the generic model was adapted. The scattering cross section was adapted from Ref. [9]. The pomeron $\left(\sigma_{P}\right)$ and regge $\left(\sigma_{R}\right)$ parts of the cross section per nucleon are (in $\mathrm{mb}$ ):

$\sigma_{R}=\frac{3}{2} \exp \left[2.17+\frac{0.147}{\gamma}-0.961 * \log \gamma\right]$,

$\sigma_{P}=4.14+1.50 \sqrt{\gamma}-0.0545 \gamma+0.000822 \gamma^{3 / 2}$.

The scattering cross sections for squark and gluino $R$ hadrons ${ }^{5}$ become:

$$
\begin{aligned}
\sigma\left(\tilde{q} \overline{q^{\prime}}\right) & =\sigma_{P}+\sigma_{R}, \\
\sigma(\tilde{q} u d) & =2 \sigma_{P}, \\
\sigma\left(\tilde{g} q \overline{q^{\prime}}\right) & =\sigma_{R}+2 \sigma_{P}, \\
\sigma(\tilde{g} u d s) & =3 \sigma_{P},
\end{aligned}
$$

\footnotetext{
${ }^{5}$ Gluino antibaryons are not considered here for several reasons. The probability of the formation of an antibaryon following the hadronisation of a gluino is predicted to be small $(\sim 1.5 \%)$ [4]. Furthermore, the probability of a gluino-based $R$-meson acquiring negative baryon number state in an interaction in matter would also be expected to be strongly suppressed.
} 


$$
\begin{aligned}
\sigma\left(\overline{\tilde{q}} q^{\prime}\right) & =\sigma_{P}, \\
\sigma(\overline{\tilde{q}} \bar{u} \bar{d}) & =2\left(\sigma_{P}+\sigma_{R}\right)+\frac{30}{\sqrt{\gamma}} .
\end{aligned}
$$

The available baryon states were chosen as shown in table 1 and the relative rates of the available processes reweighted to match the prescription in Sect. 3.1. This meant enforcing a $10 \%$ probability of exotic meson $\rightarrow$ baryon conversion as well as disabling the phase space weights of the generic model. The code is available for download [34].

Using single particle simulations, it was verified that the main features of the triple regge model were well reproduced in the GEANT-4 implementation. After the process reweighting, baryon number changing processes formed $10 \%$ of all meson interactions. The required charge exchange probabilities for meson-to-meson transformations were guaranteed by the degeneracy of the $R$-mesons and the availability of the same number and type of channels to every meson in a multiplet. It was also confirmed that the GEANT-4 version reproduces the expected energy loss and charge exchange rates.

\section{Results}

To study the expected effects of scattering on $R$-hadrons, simulations were made of the passage through iron of $R$ hadrons of $300 \mathrm{GeV}$ mass. ${ }^{6}$ The kinematic distributions of the $R$-hadrons were given by the PYTHIA generator [35] which simulated the direct pair production of $\tilde{t} \overline{\tilde{t}}, \tilde{b} \overline{\tilde{b}}$ and $\tilde{g} \tilde{g}$ in proton-proton collisions at $14 \mathrm{TeV}$ centre-of-mass energy. The process through which the partonic final state was hadronised and $R$-hadrons were formed was modelled with customised PYTHIA-routines [36] based on the Lund string model [37]. A noteworthy free parameter in this program is the probability of the formation a $\tilde{g} g$-state following the hadronisation of a gluino. This was set to the default value of 0.1 [36]. The influence of the value of this free parameter on the results shown in this section is mentioned below.

For the results shown in this section, the allowed $R$ mesons and baryons were assigned the masses prescribed in Table 1. Furthermore, unless otherwise stated, all predictions were calculated with the regge model.

Figure 3 shows the predicted average energy loss per hadronic collision for squark-based $R$-mesons. There are only small differences between the generic and triple regge models. The energy losses of squark and gluino-based $R$ baryons in both approaches (not shown) are similar to the distributions shown in Fig. 3, with differences being of size

\footnotetext{
${ }^{6}$ The conclusions of this work are unchanged if other values of $R$ hadron masses which are kinematically feasible at the LHC and not excluded by other colliders are considered.
}

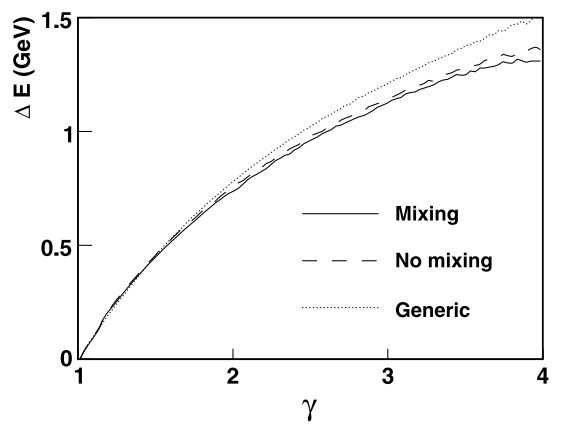

Fig. 3 Average energy loss per hadronic collision. Predictions of the generic and triple regge model are shown. The influence of mixing is also shown

$\mathcal{O} 100 \mathrm{MeV}$. Similar differences are obtained if the assumed stable states are changed from those prescribed in Table 1 to those used as default stable states with the generic model.

The total energy loss for $R$-hadrons passing through $2 \mathrm{~m}$ of iron is shown in Fig. 4(a). The length of $2 \mathrm{~m}$ was chosen since it corresponds to the typical depth of a calorimeter at a collider [38]. Gluino, stop and sbottom-based $R$-hadrons which start in the states $\tilde{t} \bar{d}^{+}, \tilde{b} \bar{u}^{-}$, and $\tilde{g} u \bar{d}^{+}$, respectively are shown.

For a zero-mixing hypothesis, the stop-based $R$-hadron suffers an average energy loss $(\sim 5.5 \mathrm{GeV})$, which is substantially greater than the energy losses for sbottom and gluino-based $R$-hadrons. This effect is due to the different $R$-mesons converting into baryonic states. Of the different stable baryon states, only the stop-based $R$-baryon is charged and therefore able to lose energy through continuous ionisation; conversions into baryon states are studied in more detail below. As can be seen in Fig. 4(a), the energy loss for the gluino-based $R$-hadron is slightly larger than that for the sbottom case owing to the typically greater number of hadronic interactions in the gluino case, as seen in Fig. 4(b). It is also shown that mixing has only a small effect on the energy loss and the number of hadronic interactions of the squark-based $R$-hadrons.

While $R$-hadron energy loss is a useful possible signature in a collider search, the charge of the $R$-hadron after passing through matter is of arguably greater importance to the development of search strategies. As mentioned earlier, this is due to searches typically looking for muon-like candidates emerging from thick calorimeter material.

In Fig. 5 the proportions of different types of gluino $R$ hadrons are shown as a function of penetration depth in iron. Prior to traversal, the proportions and kinematic properties of different hadronic states were calculated by the Lund string model in PyтHIA, as mentioned earlier. Spectra are shown for the regge and generic models in order to study the influence of the different factors which contribute to flavour composition. Furthermore, for each model, the mass hierarchies used with the regge model are used, implying that the 


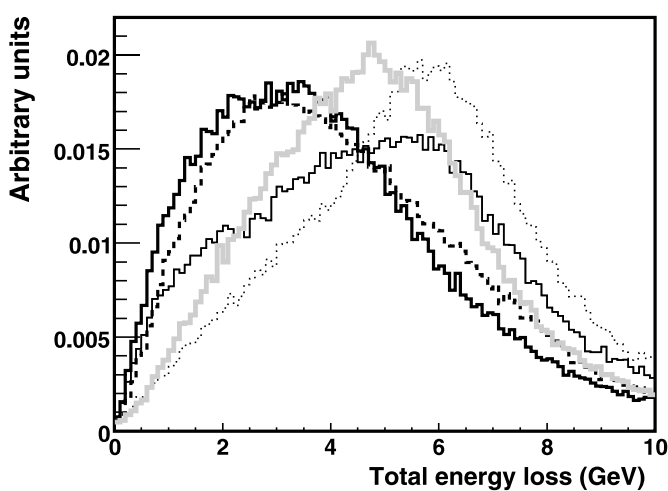

(a)

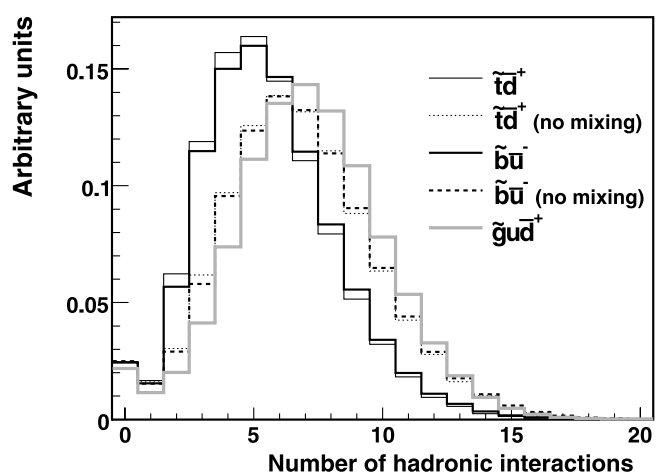

(b)

Fig. 4 Total energy loss (a) and number of hadronic interactions (b) for different types of $R$-hadrons after propagating through $2 \mathrm{~m}$ of iron

only differences are due to different approaches to scattering.

As can be seen, in both approaches, the $R$-hadrons quickly become neutral states although the rates differ. Furthermore, the spectra also show that gluino-based $R$-hadrons can reverse the sign of their charge through scattering effects. After $2 \mathrm{~m}$ of iron, small fractions of $R$-hadrons are stopped: $\sim 3 \%$ and $\sim 1 \%$ for the regge and generic models, respectively. The differences between the spectra of the generic and regge models can be qualitatively understood by considering that processes involving baryon number exchange occur at a faster rate in the generic model than the regge approach. Consequently the proportion of neutral baryonic states after $2 \mathrm{~m}$ of iron is $\sim 60 \%(\sim 100 \%)$ in the regge (generic) model. Similarly, a small fraction of gluino $R$-hadrons would be predicted to stop in the generic model since these particles are transformed into electrically neutral objects at an earlier stage.

It can be concluded that, with the assumed mass hierarchy, both models predict that the neutral states would largely be dominant after a passage through several metres of iron. The effects (not shown) of using the mass hierarchy traditionally used with the generic approach for both models would give a similar conclusion regarding the relative rates of baryon production. However, the models would not tend

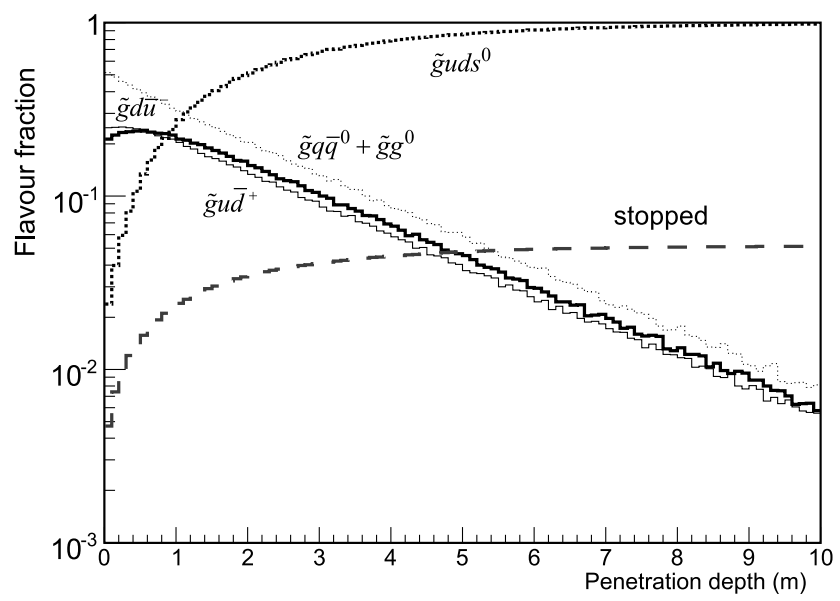

(a) Gluinos, regge model

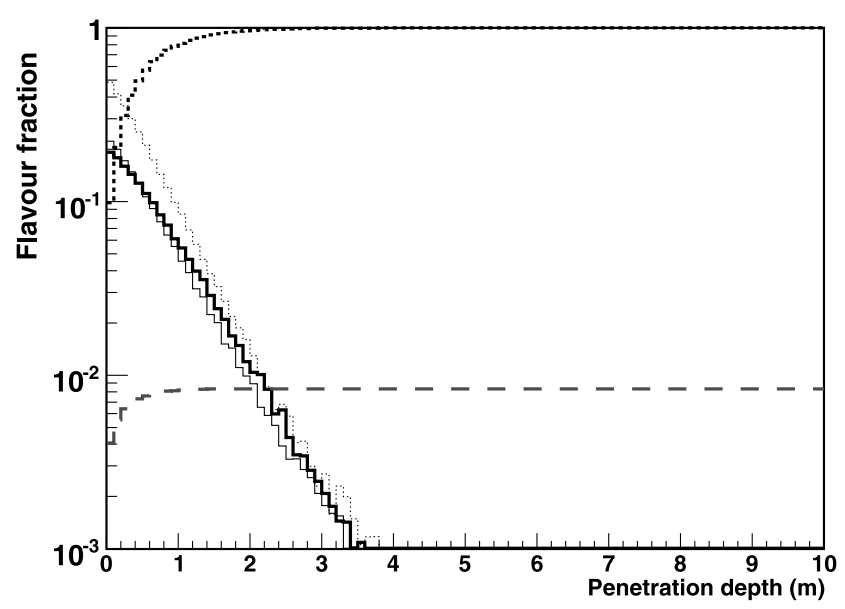

(b) Gluinos, generic model

Fig. 5 Flavour composition of $R$-hadrons as a function of penetration depth in iron. Distributions are shown for $R$-hadrons formed from gluinos. Predictions from the regge model and generic model are shown, using the assumed mass hierarchy described in Sect. 2

to predict a dominant neutral baryon state as penetration depth increases, rather an approximately equal mixture of $R^{-}(\tilde{g} d d d), R^{0}(\tilde{g} u d d), R^{+}(\tilde{g} u u d)$ and $R^{++}(\tilde{g} u u u)$ states.

As mentioned earlier, another uncertainty which could conceivably affect the fraction of neutral states after passage through matter is the proportion of $\tilde{g} g$-states produced in the hadronisation process. However, since such a state is assumed to scatter in the same way as a gluino-based $R$-meson, the abovementioned conclusions concerning the dominant neutral states would still be valid independently of the probability of a $\tilde{g} g$-state being produced in the primary interaction.

In Figs. 6(a)-6(d) similar spectra to those in Fig. 5 are shown for stop, and sbottom-based $R$-hadrons using the regge model. Again, the effects of $R$-mesons quickly transforming to baryons is apparent. $R$-hadrons formed from stop 


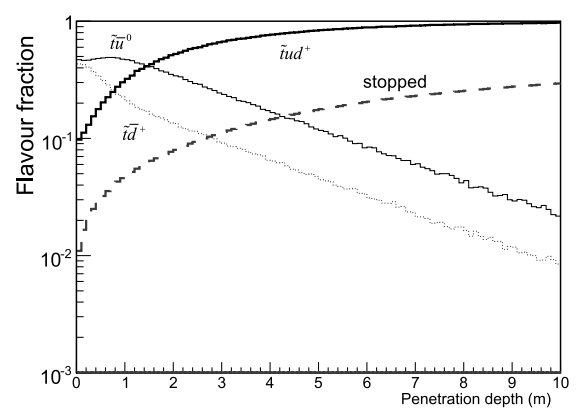

(a) Stops, no mixing

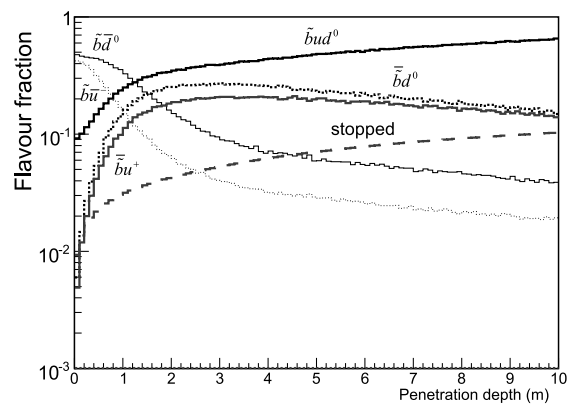

(d) Sbottoms, mixing

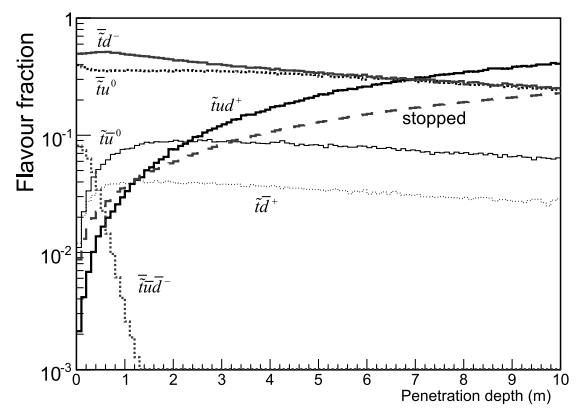

(g) Antistops, mixing

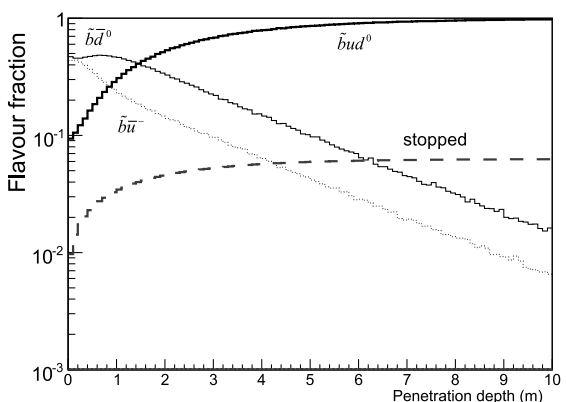

(b) Sbottoms, no mixing

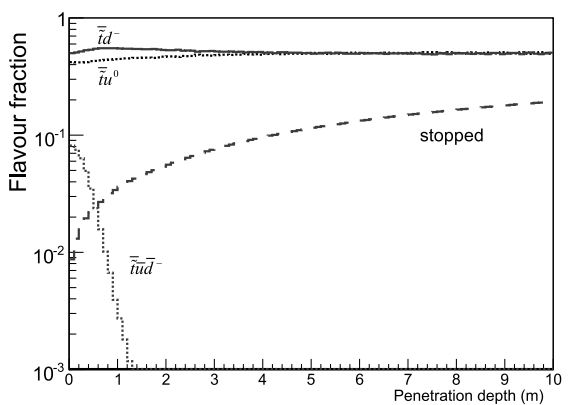

(e) Antistops, no mixing

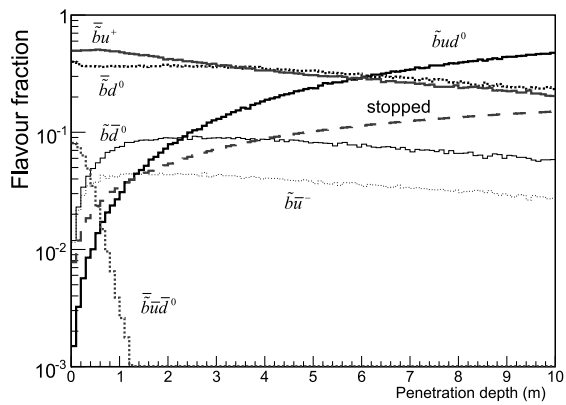

(h) Antisbottoms, mixing

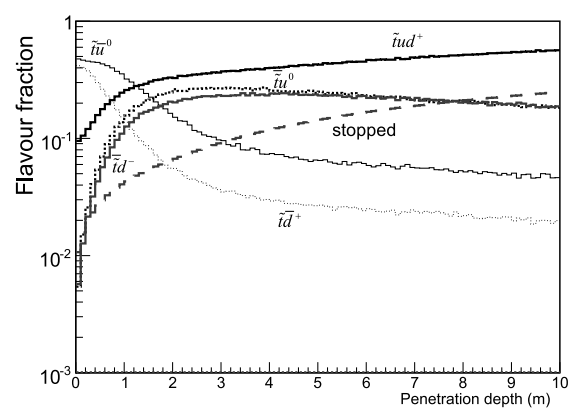

(c) Stops, mixing

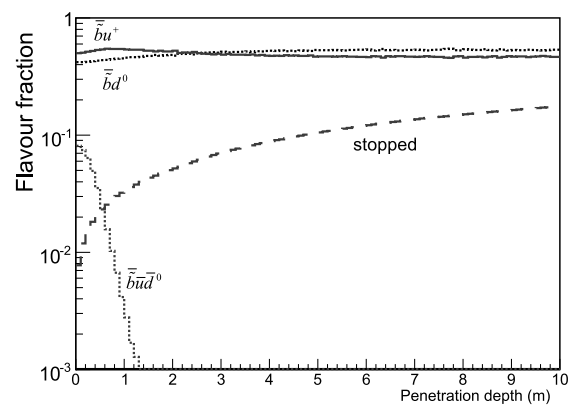

(f) Antisbottoms, no mixing

Fig. 6 Flavour composition of $R$-hadrons as a function of penetration depth in iron. Distributions made with the regge model are shown for $R$-hadrons formed from stops, sbottoms and their anti particles. The results of zero and maximal-mixing hypotheses are shown

(sbottom) squarks become largely positively charged (neutral) after several metres of iron. Of the remaining mesonic $R$-hadrons, around one half would be electrically neutral. Thus, the majority of gluino and sbottom-based $R$-hadrons would be neutral after a passage of several metres in iron. As discussed for the case of gluino $R$-hadrons, the generic model would provide qualitatively similar results albeit for a faster transformation to baryon states. Furthermore, if the stable baryons were conservatively assumed to be $\tilde{q} u d, \tilde{q} d d$, and $\tilde{q} u u$-states, then the models would asymptotically give a roughly equal mixture of these states as penetration depth increases.

The effects of maximal mixing, while tending to slow down the meson-to-baryon conversion rate, similarly do not affect the conclusion that squark-based $R$-mesons will dominantly be converted to baryons after travelling $\sim 2 \mathrm{~m}$ of iron.
It is also implied in Figs. 6(a)-6(d) that squark-based $R$ hadrons can change their charge, as for the gluino case.

For the stop-based $R$-hadrons the sign of the charge can be reversed through mixing, whereby a positively charged meson can interact to become a neutral state which oscillates into its antiparticle before interacting again to become a negatively charged meson. Also shown is the fraction of the squark-based $R$-hadrons which give up all of their energy and are stopped in material. After $2 \mathrm{~m}$ of iron, the fraction of stopped $R$-hadrons is typically $3-7 \%$, depending on the type of squark-based $R$-hadron and the mixing assumption.

Analogous flavour-composition distributions are shown in Fig. 6(e)-6(h) for antistop and antisbottom-based $R$ hadrons. As expected, the antibaryon states quickly die out owing to the large annihilation cross section and, in the absence of mixing, charged and neutral mesonic states are ex- 
pected in roughly equal proportions. The effects of mixing allow the formation of stop and sbottom-based baryons and the same trends are thus observed as in Fig. 6, albeit over longer traversal distances.

\section{Implications of $\boldsymbol{R}$-hadron scattering on searches at colliders}

Searches for stable $R$-hadrons at the Tevatron have exploited the expected penetrating muon-like behaviour of $R$ hadrons [26-30]. The most recent such search was made by the CDF experiment which reported a lower mass limit for stable stop squarks of around $250 \mathrm{GeV}$ [30]. It is, however, not trivial to use this result to estimate mass limits on stable sbottom or gluino $R$-hadrons since, as discussed in this paper, such particles could dominantly enter a muon chamber as electrically neutral objects.

There has so far been only one SMP-search [27] by a Tevatron experiment at which limits were estimated for heavy stable fermionic particles with colour octet charge. ${ }^{7}$ Under the assumption that the SMP would be electrically charged as it propagates through the detector this work excludes stable gluinos of masses less than around $200 \mathrm{GeV}$. Also using this assumption, a recent SMP-search at CDF [28] has been interpreted in Ref. [39] as providing a lower mass limit of $\sim 300 \mathrm{GeV}$.

If gluino $R$-hadrons do not behave as muon-like objects then other channels need to be investigated to estimate limits. CDF-measurements of events containing jets and missing transverse energy [40] were used to estimate a lower mass limit on stable gluinos of around $170 \mathrm{GeV}$ [39]. However, this limit may only be relevant only for cases in which the $R$-hadrons are uncharged during their complete traversal of the CDF detector since the search applies a selection criteria which removes events containing isolated high momentum tracks. Similarly, the recent D0 measurement [41] of the rate of events with the monojet and missing transverse energy signature also applies selections which may suppress $R$-hadron events; in this case via the rejection of events containing calorimeter-muon candidates.

To make a rigorous estimate of gluino $R$-hadron mass limits requires dedicated detector simulations using several scattering models to estimate the effects of the various event selections. However, in the absence of this it can nevertheless be argued that the mass limits for stable gluinos may be well below $200 \mathrm{GeV}$. In a pessimistic scenario, lower mass limits on stable gluinos would be $\sim 30 \mathrm{GeV}[42,43]$,

\footnotetext{
${ }^{7}$ The D0 experiment has looked for the late decays of long-lived gluino-based $R$-hadrons which would have stopped in it's calorimeters [14]. As for the non-decaying case, the obtained limits are sensitive to the assumptions made on the charge of $R$-hadrons in material.
}

as obtained at LEP-2. At LEP searches typically assumed the production of a substantial rate of electrically charged $R$-hadrons and information from inner tracking devices was primarily used. Similarly, the mass limits on stable sbottomlike objects would be around $90 \mathrm{GeV}[42,44]$.

Hadronic interactions and the properties of the lightest $R$-hadrons can thus play a crucial role in determining detector signatures. Given the uncertainties on the properties of $R$-hadrons both at production and during their traversal of a detector it is therefore important that LHC experiments utilise fully the capabilities of their various subdetectors to look for a number of signatures.

\section{Summary}

A model of squark-based $R$-hadron scattering, which uses triple regge phenomenology, has been implemented in GEAnT-4. The model has also been extended to include the interactions of gluino-based $R$-hadrons. The expected energy loss and the transformation of $R$-hadrons as they propagate through material was studied. The model is complementary to an existing GEANT- 4 approach for $R$-hadron scattering and will aid the development of search strategies based on a range of different signatures associated with $R$ hadron production.

The implications of $R$-hadron scattering on the results of previous searches were also discussed. In the context of the model discussed in this work, it was shown that mass limits on different types of $R$-hadrons may be lower than previously thought.

The location of the GEANT-4 code together with instructions for running it can be found in Ref. [34].

Acknowledgements The authors wish to thank Nick Ellis, Beate Heinemann, Alexei Kaidalov and Terry Sloan for helpful comments and fruitful discussions. We are also very grateful to Glennys Farrar for useful discussions regarding calculations of $R$-hadron masses made with the bag model $[19,20]$.

David Milstead is a Royal Swedish Academy Research Fellow supported by a grant from the Knut and Alice Wallenberg Foundation.

\section{References}

1. C. Amsler et al. (Particle Data Group), Phys. Lett. B 667, 1 (2008)

2. M. Drees, X. Tata, Phys. Lett. B 252, 695 (1990)

3. M.L. Perl, P.C. Kim, V. Halyo, E.R. Lee, I.T. Lee, D. Loomba, K.S. Lackner, Int. J. Mod. Phys. A 16, 2137 (2001). arXiv:hep-ex/0102033

4. M. Fairbairn, A.C. Kraan, D.A. Milstead, T. Sjöstrand, P. Skands, T. Sloan, Phys. Rep. 438, 1 (2007). arXiv:hep-ph/0611040

5. A.R. Raklev, arXiv:0908.0315 [hep-ph]

6. G. Aad et al. (The ATLAS Collaboration), CERN-OPEN-2008020. arXiv:0901.0512

7. CMS Collaboration, CMS Analysis Note: PAS EXO-08-003, 2008 
8. J.L. Pinfold (MOEDAL Collaboration), Nucl. Phys. Proc. Suppl. 78, 52 (1999)

9. Y.R. de Boer, A.B. Kaidalov, D.A. Milstead, O.I. Piskounova, J. Phys. G 35, 075009 (2008). arXiv:0710.3930 [hep-ph]

10. P.D.B. Collins, An Introduction to Regge Theory and High-Energy Physics (Cambridge, 1977), $445 \mathrm{p}$.

11. S. Agostinelli et al. (GEANT4 Collaboration), Nucl. Instrum. Methods A 506, 250 (2003)

12. L.A. Anchordoqui, A. Delgado, C.A. Garcia Canal, S.J. Sciutto, Phys. Rev. D 77, 023009 (2008). arXiv:0710.0525 [hep-ph]

13. A. Arvanitaki, S. Dimopoulos, A. Pierce, S. Rajendran, J.G. Wacker, Phys. Rev. D 76, 055007 (2007). arXiv:hep-ph/0506242

14. V.M. Abazov et al. (D0 Collaboration), Phys. Rev. Lett. 99, 131801 (2007). arXiv:0705.0306 [hep-ex]

15. R. Mackeprang, A. Rizzi, Eur. Phys. J. C 50, 353 (2007). arXiv:hep-ph/0612161

16. A.C. Kraan, Eur. Phys. J. C 37, 91 (2004). arXiv:hep-ex/0404001

17. G.R. Farrar, P. Fayet, Phys. Lett. B 76, 575 (1978)

18. M.S. Chanowitz, S.R. Sharpe, Phys. Lett. B 126, 225 (1983)

19. G.R. Farrar, Phys. Rev. Lett. 53, 1029 (1984)

20. F. Buccella, G.R. Farrar, A. Pugliese, Phys. Lett. B 153, 311 (1985)

21. M. Foster, C. Michael (UKQCD Collaboration), Phys. Rev. D 59, 094509 (1999). arXiv:hep-lat/9811010

22. N. Isgur, M.B. Wise, Phys. Lett. B 232, 113 (1989)

23. N. Isgur, M.B. Wise, Phys. Lett. B 237, 527 (1990)

24. N. Isgur, M.B. Wise, Phys. Rev. Lett. 66, 1130 (1991)

25. S.J.J. Gates, O. Lebedev, Phys. Lett. B 477, 216 (2000). arXiv:hep-ph/9912362

26. F. Abe et al. (CDF Collaboration), Phys. Rev. Lett. 63, 1447 (1989)

27. F. Abe et al. (CDF Collaboration), Phys. Rev. D 46, 1889 (1992)

28. D.E. Acosta et al. (CDF Collaboration), Phys. Rev. Lett. 90, 131801 (2003). arXiv:hep-ex/0211064
29. V.M. Abazov et al. (D0 Collaboration), Phys. Rev. Lett. 102, 161802 (2009). arXiv:0809.4472 [hep-ex]

30. T. Aaltonen et al. (CDF Collaboration), arXiv:0902.1266 [hep-ex]

31. H. Baer, K.M. Cheung, J.F. Gunion, Phys. Rev. D 59, 075002 (1999). arXiv:hep-ph/9806361

32. A. Mafi, S. Rabi, Phys. Rev. D 62, 3 (1999)

33. U. Sarid, S.D. Thomas, Phys. Rev. Lett. 85, 1178 (2000). arXiv:hep-ph/9909349

34. http://r-hadrons.web.cern.ch/r-hadrons/

35. T. Sjöstrand, S. Mrenna, P. Skands, J. High Energy Phys. 0605, 026 (2006). arXiv:hep-ph/0603175

36. The source code for the dedicated $R$-hadron hadronisation routines can be downloaded from: http://home.thep.lu.se/ torbjorn/ pythiaaux/recent.html

37. B. Andersson, G. Gustafson, G. Ingelman, T. Sjostrand, Phys. Rep. 97, 31 (1983)

38. R. Wigmans, Calorimetry: Energy Measurement in Particle Physics. Int. Ser. Monogr. Phys., vol. 107 (2000)

39. J.L. Hewett, B. Lillie, M. Masip, T.G. Rizzo, J. High Energy Phys. 0409, 070 (2004). arXiv:hep-ph/0408248

40. D. Acosta, (CDF Collaboration), Phys. Rev. Lett. 92, 121802 (2004). arXiv:hep-ex/0309051

41. V.M. Abazov et al. (D0 Collaboration), Phys. Rev. Lett. 90, 251802 (2003). arXiv:hep-ex/0302014

42. A. Heister et al. (ALEPH Collaboration), Eur. Phys. J. C 31, 327 (2003). arXiv:hep-ex/0305071

43. J. Abdallah et al. (DELPHI Collaboration), Eur. Phys. J. C 26, 505 (2003). arXiv:hep-ex/0303024

44. P. Abreu et al. (DELPHI Collaboration), Phys. Lett. B 444, 491 (1998). arXiv:hep-ex/9811007 